Pacific Journal of Mathematics

SOME REMARKS ON ACTIONS OF COMPACT MATRIX 


\section{SOME REMARKS ON ACTIONS \\ OF COMPACT MATRIX QUANTUM GROUPS ON $C^{*}$-ALGEBRAS}

Yuji Konishi, Masaru Nagisa, and Yasuo Watatani

In this paper we construct an action of a compact matrix quantum group on a Cuntz algebra or a UHF-algebra, and investigate the fixed point subalgebra of the algebra under the action. Especially we consider the action of ${ }_{\mu} U(2)$ on the Cuntz algebra $\mathscr{O}_{2}$ and the action of $S_{\mu} U(2)$ on the UHF-algebra of type $2^{\infty}$. We show that these fixed point subalgebras are generated by a sequence of Jones' projections.

1. Compact matrix quantum groups and their actions. We use the terminology introduced by Woronowicz([6]).

Definition. Let $A$ be a unital $C^{*}$-algebra and $u=\left(u_{k l}\right)_{k l} \in M_{n}(A)$, and $\mathscr{A}$ be the *-subalgebra of $A$ generated by the entries of $u$. Then $G=(A, u)$ is called a compact matrix quantum group (a compact matrix pseudogroup) if it satisfies the following three conditions:

(1) $\mathscr{A}$ is dense in $A$.

(2) There exists a *-homomorphism $\Phi$ (comultiplication) from $A$ to $A \otimes_{\alpha} A$ such that

$$
\Phi\left(u_{k l}\right)=\sum_{r=1}^{n} u_{k r} \otimes u_{r l} \quad(1 \leq k, l \leq n),
$$

where the symbol $\otimes_{\alpha}$ means the spatial $C^{*}$-tensor product.

(3) There exists a linear, antimultiplicative mapping $\kappa$ from $\mathscr{A}$ to $\mathscr{A}$ such that

$$
\kappa\left(\kappa\left(a^{*}\right)^{*}\right)=a \quad(a \in \mathscr{A})
$$

and

$$
\kappa\left(u_{k l}\right)=\left(u^{-1}\right)_{k l} \quad(1 \leq k, l \leq n) .
$$

We call $w \in B\left(C^{N}\right) \otimes A \cong M_{N} \otimes A$ a representation of a compact matrix quantum group $G=(A, u)$ on $C^{N}$ if $w \oplus w=(\operatorname{id} \otimes \Phi) w$, where $\Theta$ is a bilinear map of $\left(M_{N} \otimes A\right) \times\left(M_{N} \otimes A\right)$ to $M_{N} \otimes A \otimes A$ as follows:

$$
(l \otimes a) \oplus(m \otimes b)=l m \otimes a \otimes b
$$

for any $l, m \in M_{N}$ and $a, b \in A$. 
It is known that a compact matrix quantum group $G=(A, u)$ has the Haar measure $h$, that is, $h$ is a state on $A$ satisfying

$$
(h \otimes \mathrm{id}) \Phi(a)=(\mathrm{id} \otimes h) \Phi(a)=h(a) 1 \quad \text { for any } a \in A .
$$

So any finite dimensional representation is equivalent to a unitary representation. In this paper we only treat a unitary representation of a compact matrix quantum group.

Definition. Let $B$ be a $C^{*}$-algebra and $\pi$ be a *-homomorphism from $B$ to $B \otimes_{\alpha} A$. Then we call $\pi$ an action of a compact matrix quantum group $G=(A, u)$ on $B$ if $\left(\pi \otimes \mathrm{id}_{A}\right) \pi=\left(\mathrm{id}_{B} \otimes \Phi\right) \pi$.

Let $w$ be a unitary representation of a compact matrix quantum group $G=(A, u)$ and belong to $M_{N}(A)$. We denote by $\mathscr{O}_{N}$ the Cuntz algebra which is generated by isometries $S_{1}, \ldots, S_{N}$ satisfying $\sum_{i=1}^{N} S_{i} S_{i}^{*}=1([1])$. Then we can construct an action of $G=(A, u)$ on $\mathscr{O}_{N}$ simultaneously to [2], [3].

THEOREM 1. For a unitary representation $w \in M_{N}(A)$ of a compact matrix quantum group $G=(A, u)$, there exists an action $\varphi$ of the compact matrix quantum group $G=(A, u)$ on the Cuntz algebra $\mathscr{O}_{N}$ such that

$$
\varphi\left(S_{i}\right)=\sum_{j=1}^{N} S_{j} \otimes w_{j i} \quad \text { for any } 1 \leq i \leq N .
$$

Proof. We set $T_{i}=\varphi\left(S_{i}\right)=\sum_{j=1}^{N} S_{j} \otimes w_{j i}$ for any $i=1,2, \ldots, N$. By the relation $S_{i}^{*} S_{j}=\delta_{i j}$ and the unitarity of $w, T_{i}$ 's are isometries and $\sum_{i=1}^{N} T_{i} T_{i}^{*}=1$. So $\varphi$ can be extended to the ${ }^{*}$-homomorphism from $\mathscr{O}_{N}$ to $\mathscr{O}_{N} \otimes_{\alpha} A$. Then we have

$$
(\varphi \otimes \mathrm{id}) \varphi\left(S_{i}\right)=\sum_{j, k=1}^{N} S_{k} \otimes w_{k j} \otimes w_{j i}=(\mathrm{id} \otimes \Phi) \varphi\left(S_{i}\right)
$$

for any $1 \leq i \leq N$. This implies that $(\varphi \otimes \mathrm{id}) \varphi=(\mathrm{id} \otimes \Phi) \varphi$ on $\mathscr{O}_{N}$.

REMARK 2. Let $\varepsilon$ be a ${ }^{*}$-character from $\mathscr{A}$ to the algebra $C$ of all the complex numbers such that

$$
\varepsilon\left(u_{i j}\right)=\delta_{i j}
$$


for any $1 \leq i, j \leq n$ ([6]). If the above unitary representation $w$ belongs to $M_{N}(\mathscr{A})$, then the relation,

$$
(i d \otimes \varepsilon) \varphi=\operatorname{id}_{\mathscr{O}_{N}},
$$

holds on the dense ${ }^{*}$-subalgebra of $\mathscr{O}_{N}$ generated by $S_{1}, S_{2}, \ldots$, $S_{N}$.

We denote by $M_{N}^{K}$ the $K$-times tensor product of the $N \times N$-matrix algebra $M_{N}$, and define a canonical embedding $l$ from $M_{N}^{K}$ to $\mathscr{O}_{N}$ by

$$
l\left(e_{i_{1} j_{1}} \otimes \cdots \otimes e_{i_{K} j_{K}}\right)=S_{i_{1}} \cdots S_{i_{K}} S_{j_{K}}^{*} \cdots S_{j_{1}}^{*},
$$

where $\left\{e_{i j}\right\}_{i, j=1}^{N}$ is a system of matrix units of $M_{N}$. This embedding $l$ is compatible with the canonical inclusion of $M_{N}^{K}$ into $M_{N}^{K+1}$. We denote by $M_{N}^{\infty}$ the UHF-algebra of type $N^{\infty}$, which is obtained as the inductive limit $C^{*}$-algebra of $\left\{M_{N}^{K}\right\}_{K=1}^{\infty}$. We may consider the UHF-algebra $M_{N}^{\infty}$ as a $C^{*}$-subalgebra of $\mathscr{O}_{N}$ through the embedding.

COROLlARY 3. Let $\varphi$ be the action of a compact matrix quantum group $G=(A, u)$ on the Cuntz algebra $\mathscr{O}_{N}$ defined by the unitary representation $w \in M_{N}(A)$ as in Theorem 1 . Then the restriction $\psi$ of $\varphi$ on the UHF-algebra $M_{N}^{\infty}$ is also an action of $G=(A, u)$ on $M_{N}^{\infty}$ satisfying

$$
\begin{gathered}
\psi\left(e_{i_{1} j_{1}} \otimes \cdots \otimes e_{i_{K} j_{K}}\right)=\sum_{\substack{a_{1}, \ldots, a_{K} \\
b_{1}, \ldots, b_{K}}} e_{a_{1} b_{1}} \otimes \cdots \otimes e_{a_{K} b_{K}} \\
\otimes w_{a_{1} i_{1}} \cdots w_{a_{K} i_{K}} w_{b_{K} j_{K}}^{*} \cdots w_{b_{1} j_{1}}^{*}
\end{gathered}
$$

for any positve integer $K$.

REMARK 4. We define a bilinear map $\ominus$ of $\left(M_{N} \otimes A\right) \times\left(M_{N} \otimes A\right)$ to $M_{N} \otimes M_{N} \otimes A$ as follows:

$$
(l \otimes a) \oplus(m \otimes b)=l \otimes m \otimes a b
$$

for any $l, m \in M_{N}$ and $a, b \in A$. We denote $\overbrace{w \oplus \cdots \oplus w}^{K \text { times }}$ by $w^{K}$. Then $w^{K}$ is a unitary representation of a compact matrix quantum group $G=(A, u)$ if $w$ is a unitary representation of $G=(A, u)$. The above action $\psi$ is represented by the following form

$$
\psi(x)=w^{K}\left(x \otimes 1_{A}\right)\left(w^{K}\right)^{*} \text { for any } x \in M_{N}^{K} .
$$


So we call the action $\psi$ the product type action of $G=(A, u)$ on the UHF-algebra $M_{N}^{\infty}$.

Definition. Let $B$ be a $C^{*}$-algebra and $\pi$ be an action of a compact matrix quantum group $G=(A, u)$ on $B$. We define the fixed point subalgebra $B^{\pi}$ of $B$ by $\pi$ as follows :

$$
B^{\pi}=\left\{x \in B \mid \pi(x)=x \otimes 1_{A}\right\} .
$$

Let $\mathscr{P}_{N}$ be the dense ${ }^{*}$-subalgebra of $\mathscr{O}_{N}$ generated by $S_{1}, S_{2}, \ldots$, $S_{N}$ and $\mathscr{M}_{N}$ be the dense ${ }^{*}$-subalgebra $\bigcup_{K=1}^{\infty} M_{N}^{K}$ of $M_{N}^{\infty}$.

LEMMA 5. Let $h$ be the Haar measure on a compact matrix quantum group $G=(A, u)$, and we define $E_{\varphi}=(\mathrm{id} \otimes h) \varphi$ and $E_{\psi}=(\mathrm{id} \otimes h) \psi$. Then $E_{\varphi}$ (resp. $\left.E_{\psi}\right)$ is a projection of norm one from $\mathscr{O}_{N}$ onto $\left(\mathscr{O}_{N}\right)^{\varphi}$ (resp. from $M_{N}^{\infty}$ onto $\left.\left(M_{N}^{\infty}\right)^{\psi}\right)$ such that

$$
E_{\varphi}\left(\mathscr{P}_{N}\right) \subset \mathscr{P}_{N}, \quad E_{\psi}\left(\mathscr{M}_{N}\right) \subset \mathscr{M}_{N}
$$

Proof. Clearly $E_{\varphi}$ is a unital, completely positive map, $E_{\varphi}(x)=x$ for any $x \in\left(\mathscr{O}_{N}\right)^{\varphi}$, and $E_{\varphi}\left(\mathscr{P}_{N}\right) \subset \mathscr{P}_{N}$. By the property of the Haar measure, for any $x \in \mathscr{O}_{N}$, we have

$$
\begin{aligned}
E_{\varphi}\left(E_{\varphi}(x)\right) & =(\mathrm{id} \otimes h \otimes \mathrm{id})(\varphi \otimes \mathrm{id})(\mathrm{id} \otimes h) \varphi(x) \\
& =(\mathrm{id} \otimes h \otimes h)(\varphi \otimes \mathrm{id}) \varphi(x) \\
& =(\mathrm{id} \otimes h \otimes h)(\mathrm{id} \otimes \Phi) \varphi(x)=(\mathrm{id} \otimes(h \otimes h) \Phi) \varphi(x) \\
& =(\mathrm{id} \otimes h) \varphi(x)=E_{\varphi}(x) .
\end{aligned}
$$

So the assertion holds for $E_{\varphi}$.

Similarly the assertion also holds for $E_{\psi}$.

We can easily get the following lemma.

LEMMA 6. Let $\pi$ be an action of a compact matrix quantum group $G=(A, u)$ on $a C^{*}$-algebra $B$ and $B_{0}$ be a dense ${ }^{*}$-subalgebra of $B$. If $E$ is a projection of norm one from $B$ onto the fixed point subalgebra $B^{\pi}$ of $B$ by the action $\pi$ such that $E\left(B_{0}\right) \subset B_{0}$, then $B_{0} \cap B^{\pi}$ is dense in $B^{\pi}$.

We define a *-endomorphism $\sigma$ of $\mathscr{O}_{N}$ by $\sigma(X)=\sum_{i=1}^{N} S_{i} X S_{i}^{*}$ for any $X \in \mathscr{O}_{N}$. Then the restriction of $\sigma$ to the UHF-algebra $M_{N}^{\infty}$ of type $N^{\infty}$ satisfies that $\sigma(X)=1_{M_{N}} \otimes X$ for any $X \in M_{N}^{\infty}$. 
LEMMA 7. (1) If $X \in\left(\mathscr{O}_{N}\right)^{\varphi}$, then $\sigma(X) \in\left(\mathscr{O}_{N}\right)^{\varphi}$.

(2) If $X \in\left(M_{N}^{\infty}\right)^{\psi}$, then $\sigma(X) \in\left(M_{N}^{\infty}\right)^{\psi}$.

Proof. (1) For $X \in\left(\mathscr{O}_{N}\right)^{\varphi}$, we have

$$
\begin{aligned}
\varphi(\sigma(X)) & =\sum_{i=1}^{N} \varphi\left(S_{i} X S_{i}^{*}\right)=\sum_{i=1}^{N} \varphi\left(S_{i}\right)\left(X \otimes 1_{A}\right) \varphi\left(S_{i}\right)^{*} \\
& =\sum_{i, j, k=1}^{N} S_{j} X S_{k}^{*} \otimes u_{i j} u_{i k}^{*}=\sum_{i=1}^{N} S_{i} X S_{i}^{*} \otimes 1_{A}=\sigma(X) \otimes 1_{A} .
\end{aligned}
$$

(2) The assertion follows that $\psi$ is the restriction of $\varphi$.

2. Jones' projections and compact matrix quantum groups $S_{\mu} U(2)$ and ${ }_{\mu} U(2)$. We shall consider the actions of $S_{\mu} U(2)$ and ${ }_{\mu} U(2)$ coming from their fundamental representations.

Definition ([7]). A compact matrix quantum group $G=(A, u)$ is called $S_{\mu} U(2)$ if $A$ is the universal $C^{*}$-algebra generated by $\alpha, \gamma$ satisfying

$$
\begin{gathered}
\alpha^{*} \alpha+\gamma^{*} \gamma=1, \quad \alpha \alpha^{*}+\mu^{2} \gamma \gamma^{*}=1, \quad \gamma^{*} \gamma=\gamma \gamma^{*}, \\
\mu \gamma \alpha=\alpha \gamma, \quad \mu \gamma^{*} \alpha=\alpha \gamma^{*}, \quad \mu \alpha^{*} \gamma=\gamma \alpha^{*}, \quad \mu \alpha^{*} \gamma^{*}=\gamma^{*} \alpha^{*},
\end{gathered}
$$

where $-1 \leq \mu \leq 1$. Its fundamental representation $u$ is as follows:

$$
u=\left(\begin{array}{cc}
\alpha & -\mu \gamma^{*} \\
\gamma & \alpha^{*}
\end{array}\right) \in M_{2}(A) .
$$

The comultiplication $\Phi$ associated with $S_{\mu} U(2)$ is defined by

$$
\Phi(\alpha)=\alpha \otimes \alpha-\mu \gamma^{*} \otimes \gamma, \quad \Phi(\gamma)=\gamma \otimes \alpha+\alpha^{*} \otimes \gamma .
$$

We shall introduce the quantum $U(2)$ group ${ }_{\mu} U(2)$, which is obtained by the unitarization of the quantum GL(2) group.

Definition. A compact matrix quantum group $H=(B, v)$ is called ${ }_{\mu} U(2)$ if $B$ is the universal $C^{*}$-algebra generated by $\alpha, \gamma, D$ satisfying

$$
\begin{gathered}
D^{*} D=D D^{*}=1, \quad \alpha D=D \alpha, \quad \gamma D=D \gamma, \quad \alpha^{*} D=D \alpha^{*}, \\
\gamma^{*} D=D \gamma^{*}, \quad \alpha^{*} \alpha+\gamma^{*} \gamma=1, \quad \alpha \alpha^{*}+\mu^{2} \gamma \gamma^{*}=1, \quad \gamma^{*} \gamma=\gamma \gamma^{*}, \\
\mu \gamma \alpha=\alpha \gamma, \quad \mu \gamma^{*} \alpha=\alpha \gamma^{*}, \quad \mu \alpha^{*} \gamma=\gamma \alpha^{*}, \quad \mu \alpha^{*} \gamma^{*}=\gamma^{*} \alpha^{*},
\end{gathered}
$$


where $-1 \leq \mu \leq 1$. Its fundamental representation $v$ is as follows:

$$
v=\left(\begin{array}{cc}
\alpha & -\mu D \gamma^{*} \\
\gamma & D \alpha^{*}
\end{array}\right) \in M_{2}(B) .
$$

The comultiplication $\Psi$ associated with ${ }_{\mu} U(2)$ is defined by

$$
\begin{gathered}
\Psi(\alpha)=\alpha \otimes \alpha-\mu D \gamma^{*} \otimes \gamma, \quad \Psi(\gamma)=\gamma \otimes \alpha+D \alpha^{*} \otimes \gamma, \\
\Psi(D)=D \otimes D .
\end{gathered}
$$

REMARK 8 . The above $C^{*}$-algebra $B$ associated with the compact matrix quantum group ${ }_{\mu} U(2)=H=(B, v)$ is isomorphic to $A \otimes_{\alpha}$ $C(T)$ as a $C^{*}$-algebra, where $A$ is the $C^{*}$-algebra associated with the compact matrix quantum group $S_{\mu} U(2)=G=(A, u)$ and $C(T)$ is the algebra of all the continuous functions on the one dimensional torus $T$. The elements $\alpha$ and $\gamma$ in $H$ satisfy the same relation of $\alpha$ and $\gamma$ in $G$. But the values of the comultiplication $\Psi$ at $\alpha, \gamma \operatorname{differ}$ from ones of the comultiplication $\Phi$ at $\alpha, \gamma$.

In the rest of the paper, we fix a number $\mu \in[-1,1] \backslash\{0\}$.

We denote by $\varphi_{1}$ (resp. by $\varphi_{2}$ ) the action of the compact matrix quantum group ${ }_{\mu} U(2)=(B, v)$ (resp. $\left.S_{\mu} U(2)=(A, u)\right)$ on the Cuntz algebra $\mathscr{O}_{2}$ coming from the fundamental representation $v$ (resp. $u$ ) as in Theorem 1. We also denote $\psi_{1}$ (resp. $\left.\psi_{2}\right)$ the product type action of the compact matrix quantum group ${ }_{\mu} U(2)=(B, v)$ (resp. $S_{\mu} U(2)=(A, u)$ ) on the UHF-algebra $M_{2}^{\infty}$ of type $2^{\infty} \mathrm{com}$ ing from $v$ (resp. $u$ ) as in Corollary 3 .

From now on, we shall determine the fixed point subalgebras of the above actions.

In [8] Woronowicz defines the $4 \times 4$-matrix

$$
g=\left(\begin{array}{cccc}
1 & 0 & 0 & 0 \\
0 & 0 & \mu & 0 \\
0 & \mu & 1-\mu^{2} & 0 \\
0 & 0 & 0 & 1
\end{array}\right) \in M_{2} \otimes M_{2} \subset M_{2}^{\infty}
$$

and shows that the algebra $\left\{x \in M_{2}^{K} \mid u^{K}\left(x \otimes 1_{A}\right)=\left(x \otimes 1_{A}\right) u^{K}\right\}$ is generated by $g_{1}, g_{2}, \ldots, g_{K-1}$, where $g_{i+1}=\sigma^{i}(g)(i=0,1, \ldots$, $K-2)$.

We set

$$
e_{i}=\frac{1}{1+\mu^{2}}\left(1-g_{i}\right) \text { for any } i=1,2, \ldots, K-1,
$$


then the sequence $\left\{e_{n}\right\}_{n=1}^{\infty}$ of projections satisfies the Jones' relation

$$
e_{i} e_{i \pm 1} e_{i}=\frac{\mu^{2}}{\left(1+\mu^{2}\right)^{2}} e_{i}, \quad e_{i} e_{j}=e_{j} e_{i} \quad(\text { if }|i-j|>1) .
$$

We denote by $C^{*}\left(\left\{e_{n}\right\}_{n=1}^{\infty}\right)$ the unital $C^{*}$-algebra generated by the projections $\left\{e_{n}\right\}_{n=1}^{\infty}$.

Proposition 9. The fixed point subalgebra $\left(M_{2}^{\infty}\right)^{S_{\mu} U(2)}$ of the UHFalgebra $M_{2}^{\infty}$ by the action $\psi_{2}$ of $S_{\mu} U(2)$ is generated by the above Jones' projections $\left\{e_{n}\right\}_{n=1}^{\infty}$.

Proof. By Remark 4, $M_{2}^{K} \cap\left(M_{2}^{\infty}\right)^{\psi_{2}}=\left\{x \in M_{2}^{K} \mid u^{K}\left(x \otimes 1_{A}\right)=\right.$ $\left.\left(x \otimes 1_{A}\right) u^{K}\right\}$. So $M_{2}^{K} \cap\left(M_{2}^{\infty}\right)^{\psi_{2}}$ is generated by $e_{1}, e_{2}, \ldots, e_{K-1}$. The assertion follows from Lemma 5 and Lemma 6.

THEOREM 10. The fixed point subalgebra $\left(\mathscr{O}_{2}\right)^{\mu} \mathrm{U}(2)$ of the Cuntz algebra $\mathscr{O}_{2}$ by the action $\varphi_{1}$ of ${ }_{\mu} U(2)=(B, v)$ coincides with the fixed point subalgebra $\left(M_{2}^{\infty}\right)^{S_{\mu} U(2)}$ of the UHF-algebra $M_{2}^{\infty}$ by the action $\psi_{2}$ of $S_{\mu} U(2)=(A, u)$.

In particular,

$$
\left(\mathscr{O}_{2}\right)^{\mu} U(2)=\left(M_{2}^{\infty}\right)^{\mu} U(2)=\left(M_{2}^{\infty}\right)^{S_{\mu} U(2)}=C^{*}\left(\left\{e_{n}\right\}_{n=1}^{\infty}\right) .
$$

Proof. It is clear that $\left(\mathscr{O}_{2}\right)^{\mu} U(2) \supset .\left(M_{2}^{\infty}\right)^{\mu} U(2)$. In order to show that $\left(\mathscr{O}_{2}\right)^{\mu} U(2) \subset\left(M_{2}^{\infty}\right)^{\mu} U(2)$, it is sufficient to show that $\mathscr{P}_{2} \cap\left(\mathscr{O}_{2}\right)^{\varphi_{1}} \subset$ $\left(\mathscr{M}_{2} \cap\left(M_{2}^{\infty}\right)^{\psi_{1}}\right)$ by Lemma 5 and Lemma 6. Let $x \in \mathscr{P}_{2} \cap\left(\mathscr{O}_{2}\right)^{\varphi_{1}}$ and $\theta$ be a ${ }^{*}$-homomorphism of $B$ onto $C^{*}(D)$ such that $\theta(\alpha)=D, \theta(\gamma)=$ 0 and $\theta(D)=D^{2}$. The element $x$ has the unique representation

$$
x=\sum_{i>0}\left(S_{1}^{*}\right)^{i} A_{-i}+A_{0}+\sum_{i>0} A_{i}\left(S_{1}\right)^{i},
$$

where each $A_{i}(i=0, \pm 1, \pm 2, \ldots)$ belongs to $\mathscr{M}_{2}$ ([1]). Since $\left(\operatorname{id}_{\mathscr{O}_{2}} \otimes \theta\right) \varphi_{1}\left(S_{i}\right)=S_{i} \otimes D$ for any $i=1,2$,

$$
\begin{aligned}
x \otimes 1_{B} & =\left(\operatorname{id}_{\mathscr{O}} \otimes \theta\right) \varphi_{1}(x) \\
& =\sum_{i>0}\left(S_{1}^{*}\right)^{i} A_{-i} \otimes\left(D^{*}\right)^{i}+A_{0} \otimes 1_{B}+\sum_{i>0} A_{i}\left(S_{1}\right)^{i} \otimes D^{i} .
\end{aligned}
$$

Hence $x=A_{0} \in \mathscr{M}_{2} \cap\left(M_{2}^{\infty}\right)^{\psi_{1}}$. Therefore $\left(\mathscr{O}_{2}\right)^{\mu} U(2)=\left(M_{2}^{\infty}\right)^{\mu} U(2)$. 
We define a *-homomorphism $\eta$ of $B$ onto $A$ such that $\eta(\alpha)=$ $\alpha, \eta(\gamma)=\gamma$ and $\eta(D)=1$. Then the following diagram commutes

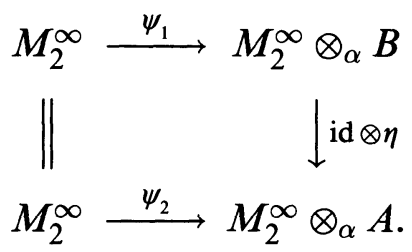

So $\left(M_{2}^{\infty}\right)^{\mu}{ }^{(2)} \subset\left(M_{2}^{\infty}\right)^{S_{\mu} U(2)}$.

We shall show that $\left(M_{2}^{\infty}\right)^{\mu} U(2) \supset\left(M_{2}^{\infty}\right)^{S_{\mu} U(2)}$. It is sufficient to show that $\left(M_{2}^{\infty}\right)^{\mu}{ }^{U(2)}$ contains $\left\{e_{n}\right\}_{n=1}^{\infty}$ by Proposition 9 . We set

$$
\tau=\left(\begin{array}{cccc}
1 & 0 & 0 & 0 \\
0 & D & 0 & 0 \\
0 & 0 & D & 0 \\
0 & 0 & 0 & D^{2}
\end{array}\right) \in M_{4}(B) \cong M_{2} \otimes M_{2} \otimes B
$$

then

$$
v \ominus v=\left(\left(\begin{array}{cc}
\alpha & -\mu \gamma^{*} \\
\gamma & \alpha^{*}
\end{array}\right) \oplus\left(\begin{array}{cc}
\alpha & -\mu \gamma^{*} \\
\gamma & \alpha^{*}
\end{array}\right)\right) \tau
$$

and

$$
\tau\left(e_{1} \otimes 1_{B}\right)=\left(e_{1} \otimes 1_{B}\right) \tau
$$

Then we have

$$
\begin{aligned}
& \psi_{1}\left(e_{1}\right)=(v \oplus v)\left(e_{1} \otimes 1_{B}\right)(v \oplus v)^{*} \\
& \quad=\left(\left(\begin{array}{cc}
\alpha & -\mu \gamma^{*} \\
\gamma & \alpha^{*}
\end{array}\right) \oplus\left(\begin{array}{cc}
\alpha & -\mu \gamma^{*} \\
\gamma & \alpha^{*}
\end{array}\right)\right) \tau\left(e_{1} \otimes 1_{B}\right) \tau^{*}\left(\left(\begin{array}{cc}
\alpha & -\mu \gamma^{*} \\
\gamma & \alpha^{*}
\end{array}\right) \oplus\left(\begin{array}{cc}
\alpha & -\mu \gamma^{*} \\
\gamma & \alpha^{*}
\end{array}\right)\right)^{*} \\
& =\left(\left(\begin{array}{cc}
\alpha & -\mu \gamma^{*} \\
\gamma & \alpha^{*}
\end{array}\right) \oplus\left(\begin{array}{cc}
\alpha & -\mu \gamma^{*} \\
\gamma & \alpha^{*}
\end{array}\right)\right)\left(e_{1} \otimes 1_{B}\right)\left(\left(\begin{array}{cc}
\alpha & -\mu \gamma^{*} \\
\gamma & \alpha^{*}
\end{array}\right) \oplus\left(\begin{array}{cc}
\alpha & -\mu \gamma^{*} \\
\gamma & \alpha^{*}
\end{array}\right)\right)^{*} \\
& =e_{1} \otimes 1_{B} .
\end{aligned}
$$

By this fact and Lemma $7, e_{n} \in\left(M_{2}^{\infty}\right)^{\mu} U(2)$ for any positive integer $n$.

So the theorem holds.

ReMARK 11. In the case $\mu=1$,

$$
e_{i} e_{i \pm 1} e_{i}=\frac{\mu^{2}}{\left(1+\mu^{2}\right)^{2}} e_{i}=\frac{1}{4} e_{i},
$$

and the projection $e_{1}$ is represented as follows:

$$
e_{1}=\frac{1}{2}\left(\begin{array}{cccc}
0 & 0 & 0 & 0 \\
0 & 1 & -1 & 0 \\
0 & -1 & 1 & 0 \\
0 & 0 & 0 & 0
\end{array}\right)
$$


Therefore the above theorem is a $C^{*}$-version of a deformation of Jones' result ([2], [4], [5]).

\section{REFERENCES}

[1] J. Cuntz, Simple $C^{*}$-algebras generated by isometries, Comm. Math. Phys., 57 (1977), 173-185.

[2] M. Enomoto and Y. Watatani, Young diagrams and $C^{*}$-algebras, Math. Japon., 25 (1980), 395-404.

[3] D. E. Evans, On $\mathscr{O}_{n}$, Publ. Res. Inst. Math. Sci., 16 (1980), 915-927.

[4] F. M. Goodman, P. de la Harpe and V. F. R. Jones, Coxeter Graphs and Towers of Algebras, Mathematical Sciences Research Institute Publications 14, Springer-Verlag.

[5] V. F. R. Jones, Index for subfactors, Invent. Math., 72 (1983), 1-25.

[6] S. L. Woronowicz, Compact matrix pseudogroups, Comm. Math. Phys., 111 (1987), 613-665.

[7] _ Twisted SU(2) group. An example of a non-commutative differential calculus, Publ. Res. Inst. Math. Sci., 23 (1987), 117-181.

[8] __ Tannaka-Krein duality for compact matrix pseudogroups. Twisted $S U(N)$ groups, Invent. Math., 93 (1988), 35-76.

Received April 4, 1990.

NiIGATA UNIVERSITY

NiIGATA 950-21, JAPAN

Chiba University

ChiBA 260, JAPAN

AND

HOKKAIDO UNIVERSITY

SAPPORO 060, JAPAN 



\section{PACIFIC JOURNAL OF MATHEMATICS EDITORS}

V. S. VARADARAJAN

(Managing Editor)

University of California

Los Angeles, CA 90024-1555-05

Herbert Clemens

University of Utah

Salt Lake City, UT 84112

F. Michael Christ

University of California

Los Angeles, CA 90024-1555

THOMAS ENRIGHT

University of California, San Diego

La Jolla, CA 92093

\section{C. MOORE}

University of California

Berkeley, CA 94720

MARTIN SCHARLEMANN

University of California

Santa Barbara, CA 93106

HAROLD STARK

University of California, San Diego

La Jolla, CA 92093

STEVEN KERCKHOFF

Stanford University

Stanford, CA 94305

ASSOCIATE EDITORS

R. ARENS

E. F. BECKENBACH (1906-1982)

B. H. NeumanN

F. WOLF

(1904-1989)

K. YosHIDA

SUPPORTING INSTITUTIONS

UNIVERSITY OF ARIZONA

UNIVERSITY OF OREGON

UNIVERSITY OF BRITISH COLUMBIA

UNIVERSITY OF SOUTHERN CALIFORNIA

CALIFORNIA INSTITUTE OF TECHNOLOGY

UNIVERSITY OF CALIFORNIA

MONTANA STATE UNIVERSITY

STANFORD UNIVERSITY

UNIVERSITY OF NEVADA, RENO

UNIVERSITY OF HAWAII

NEW MEXICO STATE UNIVERSITY

OREGON STATE UNIVERSITY

UNIVERSITY OF UTAH

WASHINGTON STATE UNIVERSITY

UNIVERSITY OF WASHINGTON 


\section{Pacific Journal of Mathematics}

\section{Vol. 153, No. $1 \quad$ March, 1992}

Patrick Robert Ahern and Carmen Cascante, Exceptional sets for Poisson integrals of potentials on the unit sphere in $\mathbf{C}^{n}, p \leq 1 \ldots \ldots \ldots \ldots \ldots$

David Peter Blecher, The standard dual of an operator space ........... 15

Patrick Gilmer, Real algebraic curves and link cobordism .............. 31

Simon M. Goberstein, On orthodox semigroups determined by their

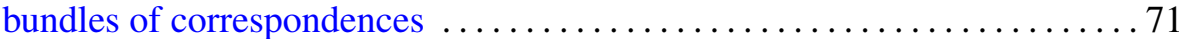

John Kalliongis and Darryl John McCullough, Homeotopy groups of irreducible 3-manifolds which may contain two-sided projective planes .......................................... 85

Yuji Konishi, Masaru Nagisa and Yasuo Watatani, Some remarks on actions of compact matrix quantum groups on $C^{*}$-algebras $\ldots \ldots \ldots \ldots 119$

Guojun Liao and Luen-Fai Tam, On the heat equation for harmonic maps from noncompact manifolds ........................... 129

John Marafino, Boundary behavior of a conformal mapping . .......... 147

Ji Min, A remark on the symmetry of solutions to nonlinear elliptic

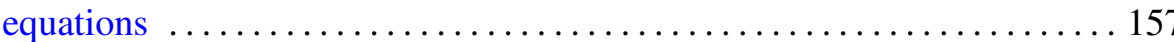

Paul Nevai and Walter Van Assche, Compact perturbations of orthogonal

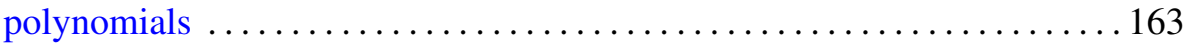

Kyril Tintarev, Level set maxima and quasilinear elliptic problems ....... 185 